\title{
Scalable Production of Iron Oxide Nanowhiskers
}

\author{
Thomas Macher, ${ }^{1}$ Jennifer Sherwood, ${ }^{1}$ Yaolin $\mathrm{Xu},{ }^{1}$ Min Lee, ${ }^{2}$ Grayson Dennis, \\ Ying Qin, ${ }^{2}$ Daniel Daly, ${ }^{2}$ Richard P. Swatloski, ${ }^{3}$ and Yuping Bao ${ }^{1}$
}

\author{
${ }^{1}$ Chemical and Biological Engineering, The University of Alabama, Tuscaloosa, AL 35487, USA \\ ${ }^{2}$ Alabama Innovation and Mentoring of Entrepreneurs, The University of Alabama, Tuscaloosa, AL 35487, USA \\ ${ }^{3}$ Office of Technical Transfer, The University of Alabama, Tuscaloosa, AL 35487, USA
}

Correspondence should be addressed to Yuping Bao; ybao@eng.ua.edu

Received 13 January 2015; Revised 13 March 2015; Accepted 16 March 2015

Academic Editor: Andrea Falqui

Copyright (C) 2015 Thomas Macher et al. This is an open access article distributed under the Creative Commons Attribution License, which permits unrestricted use, distribution, and reproduction in any medium, provided the original work is properly cited.

We reported the scalable production of iron oxide nanowhiskers from $250 \mathrm{~mL}$ laboratory scale to $10 \mathrm{~L}$ reactor reaction. During the scale-up process, the reaction time was found to be a critical parameter to adjust for the production of iron oxide nanowhiskers with similar quality to that of the laboratory scale. Based on transmission electron microscopy and magnetometry analysis, the size, size distribution, and magnetic properties of the large scale products were comparable to those produced on laboratory scale. Subsequently, these nanowhiskers were transferred from organic solvents to aqueous solution using a 38-liter stainless steel tank. These water soluble nanowhiskers were well dispersed in water and the surface chemistry was confirmed by Fourier transform infrared spectroscopy.

\section{Introduction}

Iron oxide nanoparticles have shown great potential in many applications, including contrast agents for magnetic resonance imaging (MRI) [1], iron deficiency therapy [2], drug delivery [3, 4], soil and groundwater remediation [5], and photocatalysts $[6,7]$. Iron oxide nanoparticles (magnetite or maghemite) are an inverse phase spinel crystal structure and their magnetic properties are strongly size- and shapedependent [8, 9]. Most of these applications have been focused on spherical nanoparticles with a few reported on nanocubes [10-12]. The formation of one dimensional (1D) structures was limited to iron oxide nanobars [13], nanowires [11], and iron oxide nanochains [14,15]. Recently, it was shown that nonspherical iron oxide nanoparticles could greatly improve their usefulness for biomedical applications, such as extremely high $r_{2}$ relaxivity [16] and high value of the specific absorption rate of iron oxide nanocubes [17], long blood circulation time, and enhanced retention at tumor sites of nanoworms [18]. These studies suggested that shape control of iron oxide nanoparticles could potentially lead to further advancement in biomedical applications. We and other groups have recently reported the synthesis of ultrathin $1 \mathrm{D}$ iron oxide nanostructures $[19,20]$. We recently demonstrated that these nanowhiskers can be used as novel positive contrast agents for MRI [21]. For any type of potential applications, large scale production is an important factor, such as the scaled production of spherical iron oxide nanoparticles for direct coal liquefaction [22, 23].

Here, we reported the scalable production of ultrathin iron oxide nanowhiskers using a selective heating method. The synthesis was scaled up from laboratory scale of a $250 \mathrm{~mL}$ reaction flask to a $10 \mathrm{~L}$ reactor. During this process, we discovered that the reaction time was a key parameter to be adjusted in order to achieve a similar quality product, mainly because of the alteration of heat/mass transfer rates for large volume reactions. Interestingly, the prolonged reaction time did not greatly affect the morphology and property of the nanowhiskers. The transmission electron microscopy (TEM) images showed similar morphologies of the nanowhiskers and the Fourier transform infrared spectroscopy (FTIR) spectra indicated the formation of magnetic ferrite structure. Furthermore, the phase transfer strategy from organic solvent to aqueous solution developed at laboratory scale was successfully applied to large scale (38-liter tank) to prepare water soluble iron oxide nanowhiskers. The scalability of 
the selective heating method and surface functionalization approach will open up further opportunities to explore the applications of iron oxide nanowhiskers.

\section{Experiments}

2.1. Chemicals. Reagents were purchased from VWR, unless otherwise indicated, including iron chloride $\left(\mathrm{FeCl}_{3}, \mathrm{ACROS}\right.$, 98\%), sodium oleate (TCI America, 95\%), oleylamine (TCI America, minimum 50\%), oleic acid (OA, Fisher, 95\%), trioctylphosphine oxide (TOPO, Sigma Aldrich 90\%), 1octadecene (ACROS 90\%), and polysorbate 80 (Tween 80, AMRESCO, reagent grade).

2.2. Preparation of the Iron Oleate/Oleylamine Complex. Ironligand complex precursor was prepared in a $10 \mathrm{~L}$ reactor using sodium oleate and oleylamine as mixed ligands following our previously developed procedures on laboratory scale with slight modification $[9,24-26]$. The reaction volume was first scaled up 100 times. In brief, iron chloride (140 g, $0.85 \mathrm{~mol})$, oleylamine $(289 \mathrm{~mL})$, and sodium oleate $(536 \mathrm{~g}$, $1.76 \mathrm{~mol}$ ) were reacted in a solvent mixture (deionized (DI) water, $880 \mathrm{~mL}$; hexane, $3 \mathrm{~L}$; and ethanol, $1.8 \mathrm{~L}$ ) at $60^{\circ} \mathrm{C}$ for $8 \mathrm{~h}$. After reaction, the reaction mixture was separated into two phases with organic phase on the top and aqueous phase on the bottom. The aqueous phase was drained off using the bottom drain of the reactor, and the organic phase containing the precursor was washed with DI water three times and dried under vacuum overnight. The obtained brown waxy product was then collected for subsequent characterization and nanowhisker synthesis.

2.3. Synthesis of Iron Oxide Nanowhiskers. The iron oxide nanowhiskers were synthesized in the large reactor by varying the reaction volumes. First, a scale-up reaction (100 times of the laboratory reaction scale) was performed under the same reaction conditions as laboratory scale (e.g., chemical molar ratios and reaction times); then the same reaction was performed at an increased reaction time; finally, the scale-up reaction at the full capacity of the reactor was carried out. The reaction volume of the full capacity reaction was about 450 times of the laboratory scale. In brief, iron oleate/oleylamine complex $(720 \mathrm{~g}, 1.2 \mathrm{~mol}$ ) was first dissolved in $6 \mathrm{~L}$ of $1-$ octadecene followed by the addition of a mixture of capping ligands (TOPO: $90 \mathrm{~g}, 0.225 \mathrm{~mol}$; OA: $45 \mathrm{~mL}, 0.145 \mathrm{~mol}$ ). The reaction mixture was then heated at $150^{\circ} \mathrm{C}$ for $18 \mathrm{~h}$ under inert gas (argon) blanket. After the reaction mixture was cooled down to room temperature, the nanowhiskers were cleaned with a hexane/ethanol solution and precipitated out of solution by centrifugation (Avanti J26) and dried under vacuum for $30 \mathrm{~min}$. The powder was weighed and characterized. For phase transfer, the nanowhiskers were dispersed in hexane $(50 \mathrm{mg} / \mathrm{mL})$ under sonication and stored as stock solution.

2.4. Phase Transfer Process of the Iron Oxide Nanowhiskers. The iron oxide nanowhiskers were transferred from organic solvents to aqueous solution using a second layer encapsulation method. Briefly, $5 \mathrm{~L}$ of iron oxide nanowhisker hexane solution $(1 \mathrm{mg} / \mathrm{mL})$ was mixed with $10 \mathrm{~L}$ DI water in the 38-liter stainless steel tank. After addition of $250 \mathrm{~mL}$ of polysorbate 80 (final concentration of $0.15 \mathrm{mM}$ ), the reaction mixture was sonicated for $10 \mathrm{~min}$ using a high powered sonicator (Q1375 with booster probe, $20 \mathrm{kHz}$ ). The resulting solution was kept under vacuum overnight to completely evaporate the organic solvent. Finally, light brown nanowhiskers water solution was obtained, which are stable for months without any noticeable precipitation.

2.5. Characterization. The formation of the iron-ligand complex precursor and the inverse phase spinel structure of the iron oxide nanowhiskers were confirmed by FTIR [19, 27]. The samples were prepared by mixing powder sample with $\mathrm{KBr}$ to form a $\mathrm{KBr}$ pallet and then measured on a PerkinElmer Spectrum 100 FT-IR spectrometer (Bucks, UK), equipped with an attenuated total reflectance (ATR) cell by accumulation of 4 scans, with a resolution of $2 \mathrm{~cm}^{-1}$. The size and morphology of the ultrathin nanowhiskers were examined on a FEI Tecnai F-20 TEM. The magnetic property of the nanowhiskers in dry powder form was tested by alternating gradient magnetometer (AGM) at room temperature. The hydrodynamic size and size distribution of the iron oxide nanowhiskers in aqueous solution were measured by using a Zetasizer nanoseries dynamic light scattering (DLS) to ensure uniform size distribution.

\section{Results and Discussions}

The synthesis of ultrathin iron oxide nanowhiskers was originally developed on laboratory scale using a selective heating method [19]. The entire synthetic process involves preparation of the precursor, iron-ligand complex, synthesis of ultrathin iron oxide nanowhiskers, and subsequent phase transfer from organic solvent to aqueous solution. Here, the precursor preparation and nanowhisker synthesis were scaled up to $10 \mathrm{~L}$ reactors and the phase transfer was carried out in a 38-liter tank. Figure 1 shows the experimental setup of the $10 \mathrm{~L}$ reactors and the 38 -liter stainless steel tank. The $10 \mathrm{~L}$ reactors for precursor preparation and nanowhisker synthesis are identical, which are equipped with a digitally controlled and programmable heater in a temperature range of 20$1000^{\circ} \mathrm{C}$. The 0.5 horse power (HP) motor with attached stir rod allows continuous agitation of up to $15 \mathrm{~L}$ at a constant rate of $200 \mathrm{RPM}$ to ensure full mixing and effective heat transfer. The see-through glass reactor also makes it easy to monitor the reactions. Additionally, the bottom drain assures the easy recovery of the product after reaction with almost no loss of product due to transfer or spills. The removable motor and reactor lids allow easy cleanup after reaction.

The 38-liter surface functionalization tank contains an agitator for mixing, a vacuum control system, and a bottom drain valve for easy product collection. The tank also has an airtight function, allowing the reaction to take place in a chemically confined environment. After the reaction, the product recovery can be achieved by placing a magnetic collector near the bottom drain while draining the solvents. 


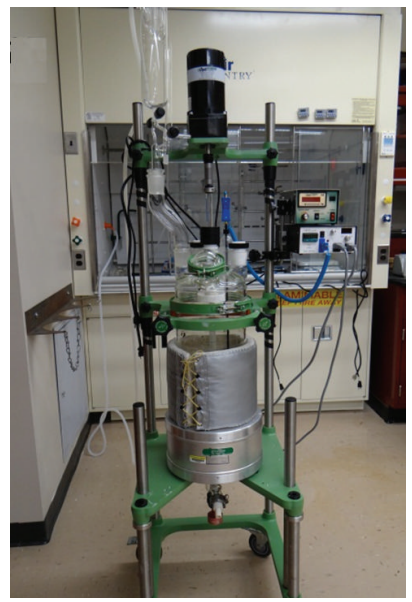

(a)

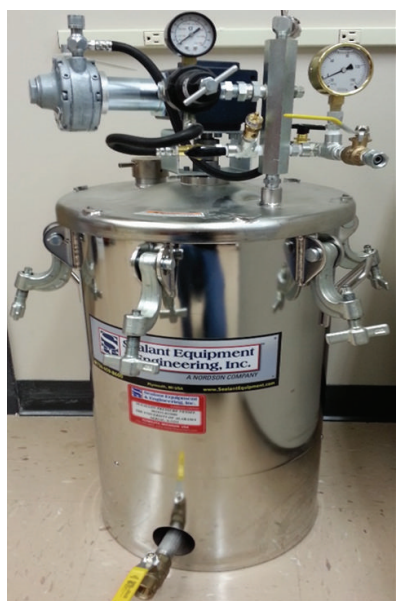

(b)
FIGURE 1: Equipment for large scale production of ultrathin nanowhiskers: (a) a picture of $10 \mathrm{~L}$ reactors for precursor preparation and nanowhisker synthesis, and (b) a picture of 38-liter stainless steel tank for phase transfer.

Cleaning the tank can be done following the same procedure used for our reactors; clean with solution and flush with water.

Using this reactor, iron-ligand complex precursor was successfully prepared with a rough yield of $94 \%$ with the production of $160 \mathrm{~g}$ per batch. The iron-ligand complex has three coordination ligands, but at this selective temperature, two of the weakly bounded ligands decompose first, leaving the third one to direct the formation of the iron oxide whiskers. Here, the modification was the addition of oleylamine as a coligand during precursor preparation with oleate to oleylamine ratio of 2 to 1 . We have previously shown that the amine functional group had a stronger binding to iron atom. The design was that the two oleate ligands will decompose first, leaving the tightly bounded oleylamine as a soft template for nanowhisker formation, which potentially allows for better control of the reaction. Compared to our previous data, this modification allowed producing iron oxide nanowhiskers with a more uniform diameter and length. The appearance and morphology of the precursor were very similar to that synthesized on laboratory scale (Figures 2(a) and 2(b)). The chemical structure of the precursors was analyzed using FTIR spectroscopy, which is a great tool to study the metal-ligand coordination. The spectra of the small scale and large scale samples were almost identical (Figure 2(c)).

The presence of the oleylamine ligand was confirmed by the broad $-\mathrm{NH}_{2}$ absorption band at $3400 \mathrm{~cm}^{-1}$ for both samples. The oleate coordination was evident from the asymmetric and symmetric $\mathrm{COO}^{-}$vibration at 1530 and $1430 \mathrm{~cm}^{-1}$. Further, the frequency difference of $100 \mathrm{~cm}^{-1}$ between the asymmetrical $\left(\nu_{\mathrm{as}}-1530 \mathrm{~cm}^{-1}\right)$ and symmetrical $\left(\nu_{\mathrm{s}}-1430 \mathrm{~cm}^{-1}\right) \mathrm{COO}^{-}$vibration models indicated the formation of bidentate coordination $[28,29]$. Compared to the large scale sample, the signal of the asymmetric $\mathrm{COO}^{-}$vibration at $1530 \mathrm{~cm}^{-1}$ was slightly higher; we attributed this to partial contribution of $-\mathrm{COOH}$ groups. This is consistent with the higher $-\mathrm{C}=\mathrm{O}$ vibration at $1713 \mathrm{~cm}^{-1}$ for the small scale sample. The bands at lower frequency $\left(690\right.$ and $\left.430 \mathrm{~cm}^{-1}\right)$ are related to the iron-oxygen coordination bands [27, 29], where the $690 \mathrm{~cm}^{-1}$ bands refer to the $\mathrm{Fe}-\mathrm{O}$ vibration with tetrahedral coordination and $430 \mathrm{~cm}^{-1}$ with octahedral coordination. The similar morphology and chemical structure of the iron complex precursors from small and large scales suggested that the scale-up process did not alter the quality of the product and further scale-up is highly feasible.

Using the precursors produced from the $10 \mathrm{~L}$ large reactor, the nanowhisker synthesis was performed in a $10 \mathrm{~L}$ reactor at various experimental conditions. The first reaction was conducted under the same reaction conditions (e.g., chemical molar ratios and reaction times) as laboratory scale, but the reaction volume was increased 100 times. The TEM image of the product showed very dark background, which is likely from the unreacted precursors (Figure 3(a)), indicating that the reaction was not complete. By keeping all the experimental parameters the same and simply increasing the reaction time from 2.5 to $6 \mathrm{~h}$, uniform iron oxide nanowhiskers were obtained (Figure 3(b)). When increasing the reaction volume to the full capacity of the reactor (about 450 times of the laboratory volume), it took about 18 hours for all the precursors to decompose and obtain uniform iron oxide nanowhiskers. The full capacity reaction can generate about $60 \mathrm{~g}$ of nanowhiskers per batch and the prolonged heating did not alter the morphology of the product (Figure 3(c)). The high resolution TEM image of the nanowhiskers from the full capacity reaction indicated their crystalline crystal structures (Figure 3(d)). Compared to the laboratory scale reaction, the reaction time was the only parameter to be adjusted to ensure completion of the reaction. The need of the larger reaction time can be understood due to the change in heat and mass transfer properties from the addition of more reactants.

The magnetic property is one of the key parameters to evaluate a magnetic material for their potential applications. The room temperature magnetization versus applied magnetic field $(\mathrm{M}-\mathrm{H})$ curve of the nanowhiskers from large scale was similar to the laboratory scale samples, but more paramagnetic, as shown in Figure 4(a). These nanowhiskers exhibited strong paramagnetic signals, indicated by the straight lines of the $\mathrm{M}-\mathrm{H}$ curves (Figure 4(a)). The high paramagnetic signal is due to the high surface to volume $(s / v)$ ratios of these nanowhiskers.

Both surface capping molecules and spin canting contributed to the paramagnetic signal. The functional groups of the ligands cause misaligned spins at the particle surface because of the broken exchange bonds from the reduced coordination of Fe surface ions while the spin-canting effects were because of the presence of disordered Fe vacancies. Surface spin-canting effects for nanostructures of high $s / v$ ratios are significantly enhanced to minimize the magnetostatic energy at the surface [30]. The canted spins on the surface of a nanostructure with high $s / v$ ratios do not respond to the external fields as bulk spins and significantly reduce the magnetization. The slight difference in the $\mathrm{M}-\mathrm{H}$ curves between small and large scale samples was attributed to the diameter uniformity of the nanowhiskers. Compared to the small scale sample, the diameter of the large scale 


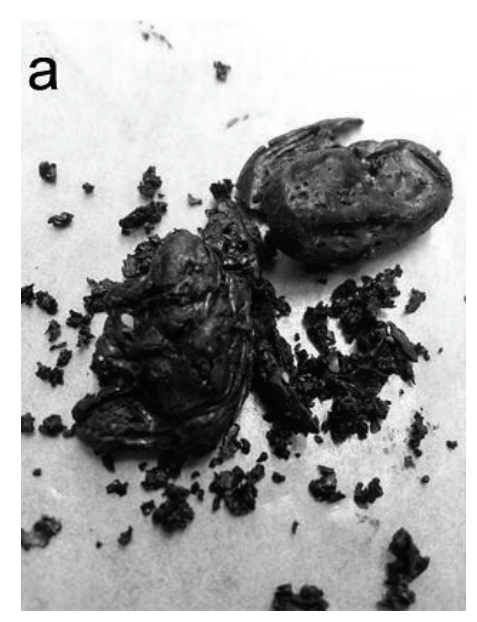

(a)

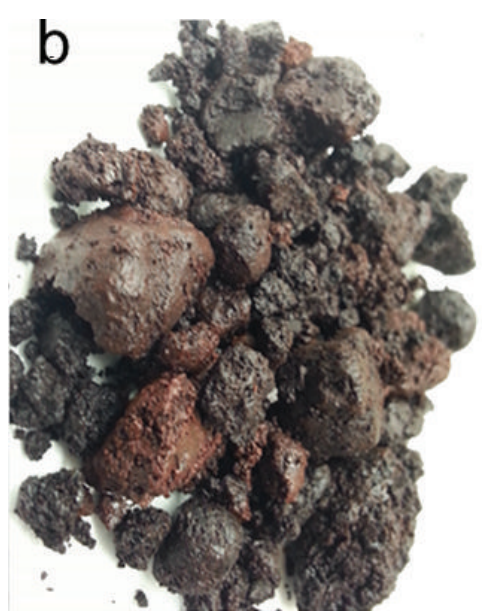

(b)

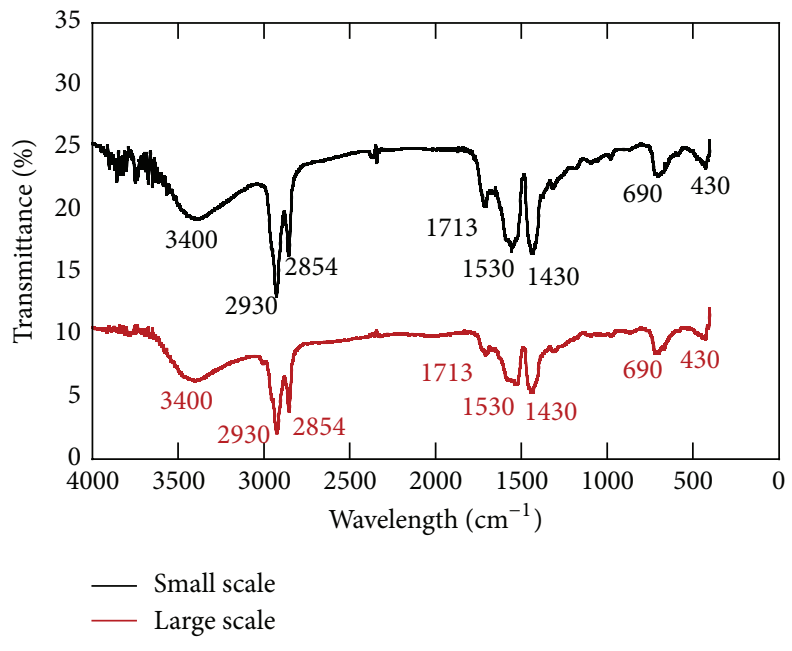

(c)

FIGURE 2: Iron oleate precursors: (a) a photograph of precursor from laboratory scale, (b) a photograph of precursor from large scale, and (c) FTIR spectra of precursors from laboratory and large scales.

sample is more uniform without thicker regions as in small scale sample. The small percentage of the superparamagnetic signal mainly resulted from these larger diameter regions. For certain application, paramagnetic signal is more preferred, such as the use of positive MRI contrast agents in our recent publications [21].

The FTIR spectra of the nanowhiskers from small and large scales both showed the typical absorption bands of spinel structures around 590 and $445 \mathrm{~cm}^{-1}$, where the $590 \mathrm{~cm}^{-1}$ band refers to the $\mathrm{Fe}-\mathrm{O}$ vibration in the tetrahedral (A) site and $445 \mathrm{~cm}^{-1}$ was assigned to the $\mathrm{Fe}-$ $\mathrm{O}$ vibration in the octahedral (B) sites. The carboxylic groups on the nanoparticle surface showed an asymmetric $\left(\mathrm{COO}^{-}\right)$vibration at $1533 \mathrm{~cm}^{-1}$ and symmetric vibration of $\left(\mathrm{COO}^{-}\right)$vibration at $1436 \mathrm{~cm}^{-1}$. The frequency difference of $97 \mathrm{~cm}^{-1}$ between the asymmetric and symmetric absorption bands suggested bidentate binding of carboxylic groups on nanowhiskers surfaces. Because of the bidentate binding (two oxygen atoms to one iron atom) and significant amount of surface atoms of the nanowhiskers, the $\mathrm{Fe}-\mathrm{O}$ band frequency shifts to higher frequency compared to typical spinel absorption bands. The chemical nature of the large scale sample was also very similar to laboratory scale sample with a frequency difference of $99 \mathrm{~cm}^{-1}$. Both samples showed the broad absorption band of $-\mathrm{NH}_{2}$ groups around $3440 \mathrm{~cm}^{-1}$, suggesting the presence of oleylamine on the nanowhisker surfaces. Samples from both laboratory scale and large scale were capped with mixed ligands oleate and oleylamine. Therefore, we believe that the scaling process did not affect the synthetic process except for the increased reaction times. For example, the reaction time was increased for the precursor preparation from laboratory scale $(4 \mathrm{~h})$ to $10 \mathrm{~L}$ reactor $(8 \mathrm{~h})$ without any noticeable change to the properties of the nanowhiskers.

The as-synthesized nanowhiskers are capped with oleate and oleylamine and only soluble in organic solvents; they must be transferred into aqueous solution for biological or biomedical applications. The mixed ligand coated iron oxide nanowhiskers were transferred into aqueous solution through a second layer encapsulation approach [31]. 


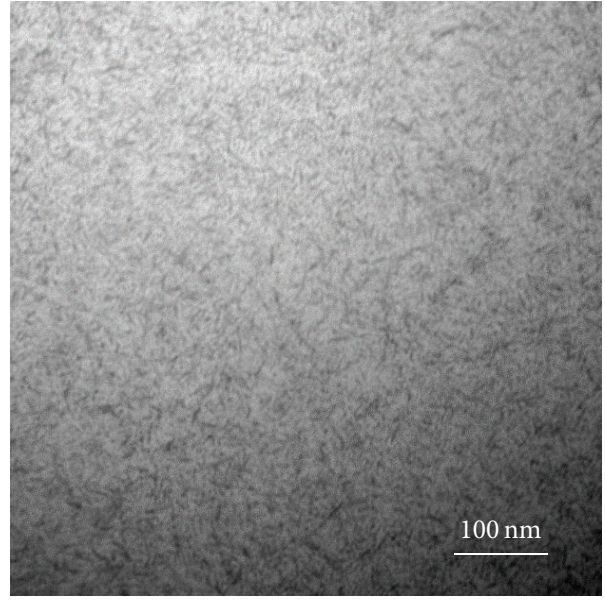

(a)

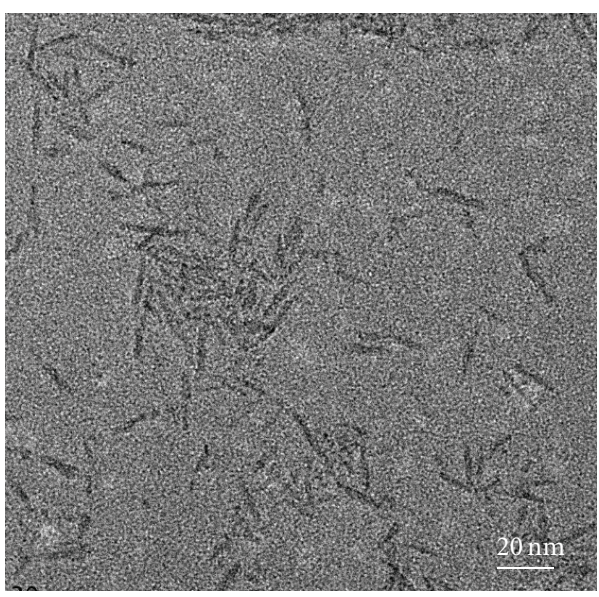

(c)

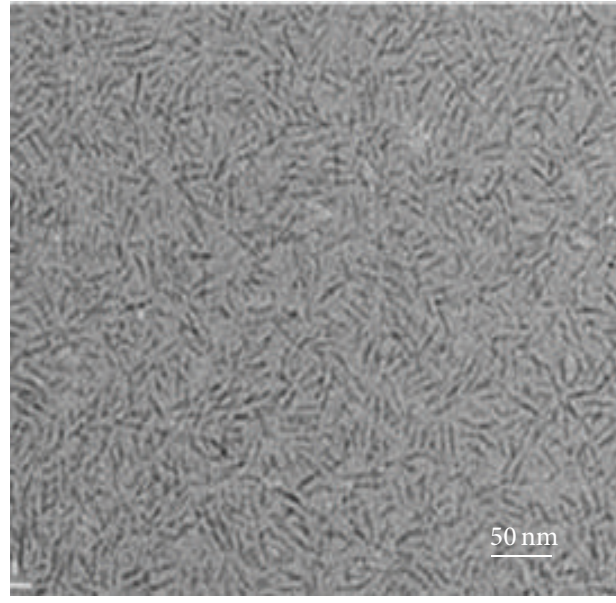

(b)

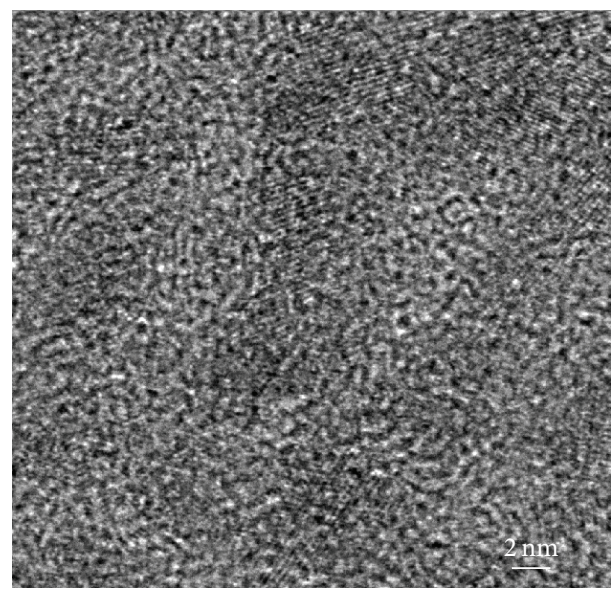

(d)

FIGURE 3: Ultrathin nanowhiskers from large scale reactions at various reaction conditions: (a) the same experimental parameters as laboratory scale but 100 times in reaction volume, (b) the same reaction condition as (a), but with an increased reaction time from 2.5 to $6 \mathrm{~h},(\mathrm{c}) 450$ times in reaction volume and $18 \mathrm{~h}$ reaction time, and (d) high resolution TEM image of image (c).

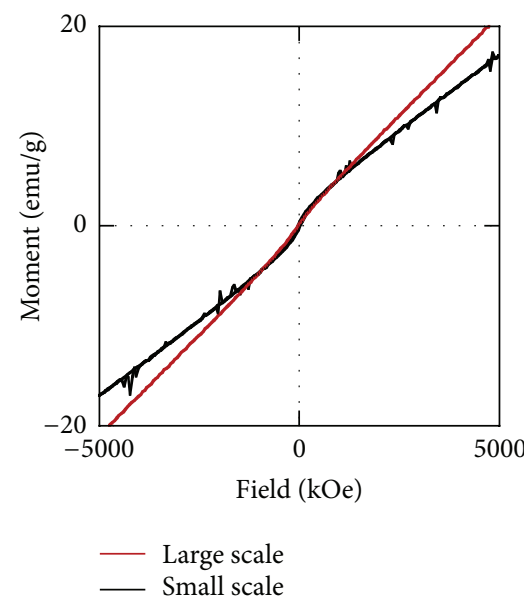

(a)

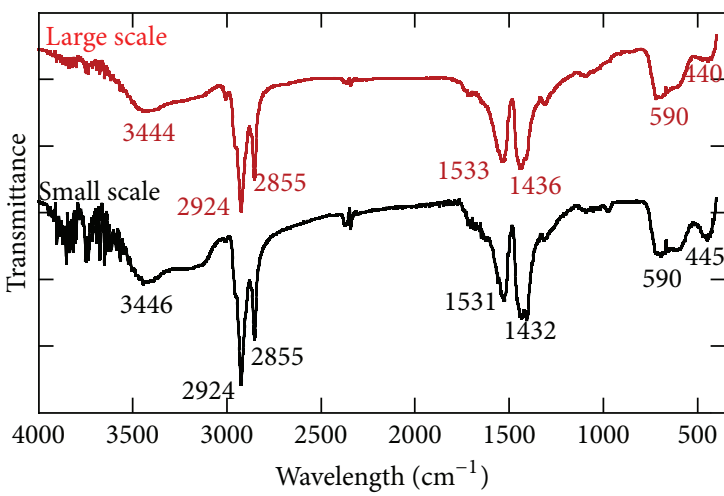

(b)

FIGURE 4: Iron oxide nanowhiskers from small and large scales: (a) M-H curves and (b) FTIR spectra. 


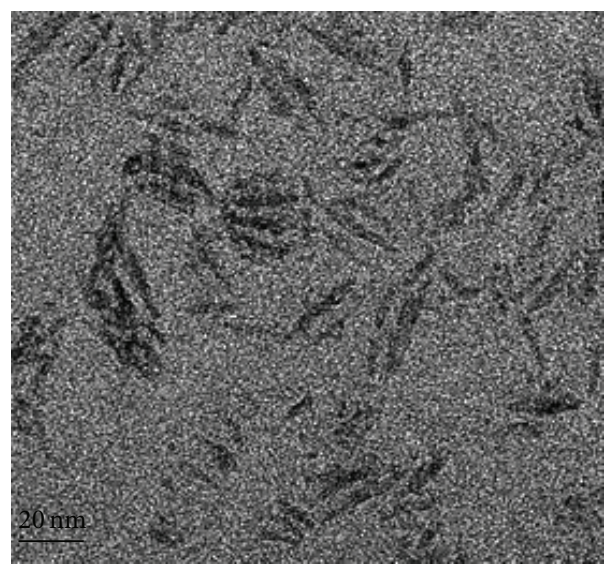

(a)

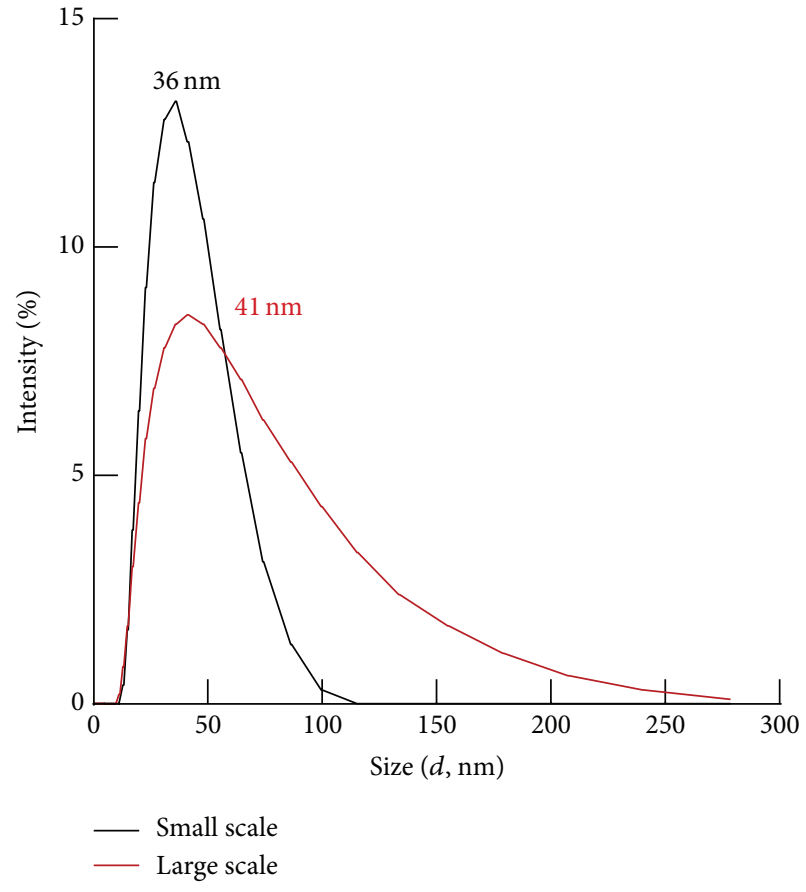

(b)

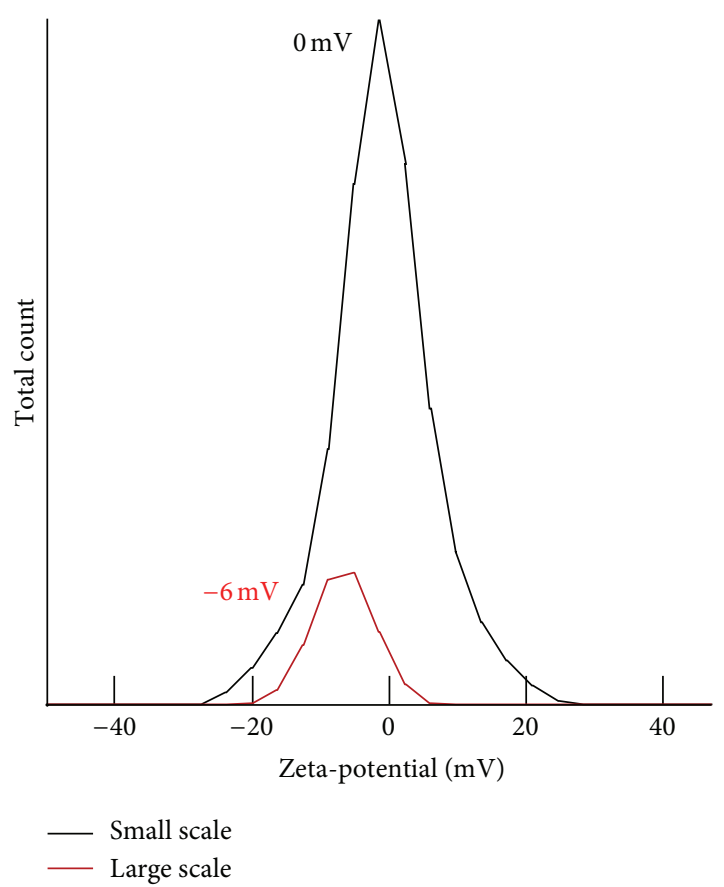

(c)

FIGURE 5: Water soluble iron oxide nanowhiskers: (a) TEM image, (b) DLS plots, and (c) zeta-potential plots.

The capping molecule for second layer encapsulation was polysorbate 80 (Tween 80 ), an amphiphilic biocompatible polymer. During mixing under sonication, the hydrophobic region of Tween 80 interacts with the hydrophobic tail of the original capping molecules on the nanowhisker surfaces, leaving the ethylene oxide polymers exposed for water solubility and biocompatibility. Before encapsulation, DLS plots of nanowhiskers in toluene were routinely measured to ensure the solution was free of aggregation. The excess Tween 80 molecules were removed by $4 \mathrm{~h}$ dialysis. Figure 5(a) shows the TEM image of Tween 80 coated iron oxide nanowhiskers from a typical large scale reaction. After phase transfer and dialysis, the nanowhiskers were well dispersed based on the TEM image. However, the DLS plot in Figure 5(b) did show an elongated tail compared to the laboratory scale sample. The long tail indicated some aggregation of the nanowhiskers by simply increasing the exchange volume. Experimental parameters need to be adjusted accordingly to achieve welldispersed samples, such as sonication power, time, or Tween 80 concentration. The zeta-potential of large scale sample showed slightly more negatively charged compared to the laboratory scale sample.

The surface chemistry of the Tween 80 coated iron oxide nanowhiskers produced at large scale was studied using FTIR (Figure 6). The spectrum showed typical stretching bands of $-\mathrm{C}-\mathrm{O}-\mathrm{C}\left(1115 \mathrm{~cm}^{-1}\right)$ and $1735 \mathrm{~cm}^{-1}$ band from $-\mathrm{C}=\mathrm{O}$ stretching of the ester bond from hydrophilic portion of Tween 80 . The much broader band around 3444 likely included the contribution from the $-\mathrm{OH}$ group of Tween 80 and the $-\mathrm{NH}_{2}$ group from the original oleylamine coating. The asymmetric $\left(1644 \mathrm{~cm}^{-1}\right)$ and symmetric $\left(1462 \mathrm{~cm}^{-1}\right)$ vibration of $\mathrm{COO}^{-}$ groups could be assigned to the original oleic acid coating. 


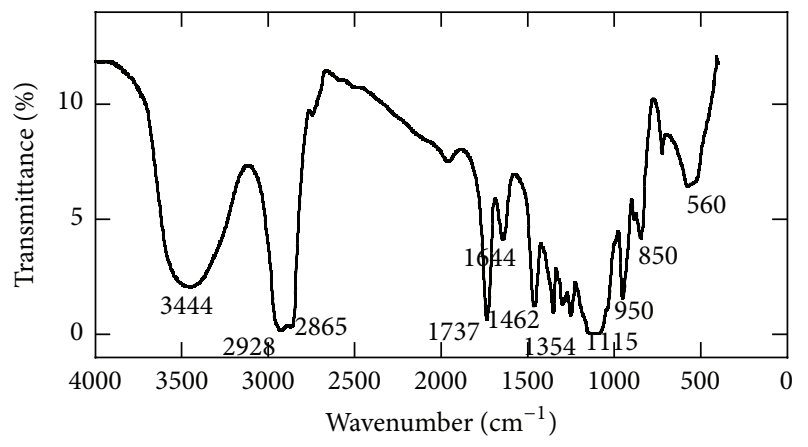

FIgURE 6: FTIR spectrum of Tween 80 coated iron oxide nanowhiskers produced at large scale.

The iron oxide vibration became one broad band at $560 \mathrm{~cm}^{-1}$. This study confirms the presence of Tween 80 on the nanowhisker surfaces. The water soluble nanowhiskers are stable for months without evident precipitations.

\section{Conclusion}

In conclusion, ultrathin iron oxide nanowhiskers were successfully prepared using $10 \mathrm{~L}$ reactors. The quality of the nanowhiskers, in terms of size, size distribution, and magnetic properties, is comparable to the samples synthesized at laboratory scale. During the scale-up process, the reaction time was found to be a critical parameter to adjust, mainly because of the slower material transfer at the large volumes. Further, the nanowhiskers were transferred from organic solvent to aqueous solution using Tween 80 as a capping molecule followed by a second layer encapsulation method. This reported scale-up study offers valuable information for future pilot studies.

\section{Conflict of Interests}

The authors declare that there is no conflict of interests regarding the publication of this paper.

\section{Acknowledgments}

This work is in part supported by DMR1149931 and NSF SBIR1248947. The authors acknowledge the UA Central Analytical Facility (CAF) and the Biological Science Department for the use of TEM. The authors would like to acknowledge the support provided by UA's Office for Technology Transfer within the Office of the Vice President for Research and Alabama Innovation and Mentoring of Entrepreneurs Center at the University of Alabama.

\section{References}

[1] P. Reimer and T. Balzer, "Ferucarbotran (Resovist): a new clinically approved RES-specific contrast agent for contrastenhanced MRI of the liver: properties, clinical development, and applications," European Radiology, vol. 13, no. 6, pp. 12661276, 2003.
[2] M. Lu, M. H. Cohen, D. Rieves, and R. Pazdur, "FDA report: ferumoxytol for intravenous iron therapy in adult patients with chronic kidney disease," The American Journal of Hematology, vol. 85, no. 5, pp. 315-319, 2010.

[3] J. Xie, J. Huang, X. Li, S. Sun, and X. Chen, "Iron oxide nanoparticle platform for biomedical applications," Current Medicinal Chemistry, vol. 16, no. 10, pp. 1278-1294, 2009.

[4] M. Namdeo, S. Saxena, R. Tankhiwale, M. Bajpai, Y. M. Mohan, and S. K. Bajpai, "Magnetic nanoparticles for drug delivery applications," Journal of Nanoscience and Nanotechnology, vol. 8, no. 7, pp. 3247-3271, 2008.

[5] H. J. Shipley, K. E. Engates, and A. M. Guettner, "Study of iron oxide nanoparticles in soil for remediation of arsenic," Journal of Nanoparticle Research, vol. 13, no. 6, pp. 2387-2397, 2011.

[6] S. Bakardjieva, V. Stengl, J. Subrt, V. Houskova, and P. Kalenda, "Photocatalytic efficiency of iron oxides: degradation of 4chlorophenol," Journal of Physics and Chemistry of Solids, vol. 68, no. 5-6, pp. 721-724, 2007.

[7] M. H. Khedr, K. S. Abdel Halim, and N. K. Soliman, "Synthesis and photocatalytic activity of nano-sized iron oxides," Materials Letters, vol. 63, no. 6-7, pp. 598-601, 2009.

[8] Q. Song and Z. J. Zhang, "Shape control and associated magnetic properties of spinel cobalt ferrite nanocrystals," Journal of the American Chemical Society, vol. 126, no. 19, pp. 6164-6168, 2004.

[9] S. Palchoudhury, Y. L. Xu, A. Rushdi, R. A. Holler, and Y. P. Bao, "Controlled synthesis of iron oxide nanoplates and nanoflowers," Chemical Communications, vol. 48, no. 85, pp. 10499-10501, 2012.

[10] D. Kim, N. Lee, M. Park, B. H. Kim, K. An, and T. Hyeon, "Synthesis of uniform ferrimagnetic magnetite nanocubes," Journal of the American Chemical Society, vol. 131, no. 2, pp. 454455, 2009.

[11] Y. Wang and H. Yang, "Synthesis of iron oxide nanorods and nanocubes in an imidazolium ionic liquid," Chemical Engineering Journal, vol. 147, no. 1, pp. 71-78, 2009.

[12] L. Y. Chen and J. Zhang, "Development of hydrophilic iron oxide nanocubes," Science of Advanced Materials, vol. 4, no. 8, pp. 859-862, 2012.

[13] C. M. Lee, H. J. Jeong, S. T. Lim, M. H. Sohn, and D. W. Kim, "Synthesis of iron oxide nanoparticles with control over shape using imidazolium-based ionic liquids," ACS Applied Materials and Interfaces, vol. 2, no. 3, pp. 756-759, 2010.

[14] Q. L. He, T. T. Yuan, S. Y. Wei et al., "Morphology- and phasecontrolled iron oxide nanoparticles stabilized with maleic anhydride grafted polypropylene," Angewandte ChemieInternational Edition, vol. 51, no. 35, pp. 8842-8845, 2012.

[15] Q. L. He, T. T. Yuan, X. R. Yan et al., "One-pot synthesis of sizeand morphology-controlled 1-D iron oxide nanochains with manipulated magnetic properties," Chemical Communications, vol. 50, no. 2, pp. 201-203, 2014.

[16] N. Lee, Y. Choi, Y. Lee et al., "Water-dispersible ferrimagnetic iron oxide nanocubes with extremely high $r_{2}$ relaxivity for highly sensitive in vivo MRI of tumors," Nano Letters, vol. 12, no. 6, pp. 3127-3131, 2012.

[17] P. Guardia, R. Di Corato, L. Lartigue et al., "Water-soluble iron oxide nanocubes with high values of specific absorption rate for cancer cell hyperthermia treatment," ACS Nano, vol. 6, no. 4, pp. 3080-3091, 2012.

[18] J.-H. Park, G. von Maltzahn, L. L. Zhang et al., "Magnetic iron oxide nanoworms for tumor targeting and imaging," Advanced Materials, vol. 20, no. 9, pp. 1630-1635, 2008. 
[19] S. Palchoudhury, W. An, Y. L. Xu et al., "Synthesis and growth mechanism of iron oxide nanowhiskers," Nano Letters, vol. 11, no. 3, pp. 1141-1146, 2011.

[20] L. Bao, W.-L. Low, J. Jiang, and J. Y. Ying, "Colloidal synthesis of magnetic nanorods with tunable aspect ratios," Journal of Materials Chemistry, vol. 22, no. 15, pp. 7117-7120, 2012.

[21] T. Macher, J. Totenhagen, J. Sherwood et al., "Ultrathin iron oxide nanowhiskers as positive contrast agents for magnetic resonance imaging," Advanced Functional Materials, vol. 25, no. 3, pp. 490-494, 2015.

[22] Y. Z. Li, F. Y. Ma, X. T. Su et al., "Ultra-large-scale synthesis of $\mathrm{Fe}_{3} \mathrm{O}_{4}$ nanoparticles and their application for direct coal liquefaction," Industrial and Engineering Chemistry Research, vol. 53, no. 16, pp. 6718-6722, 2014.

[23] Y. V. Kolen'Ko, M. Bañobre-López, C. Rodríguez-Abreu et al., "Large-scale synthesis of colloidal $\mathrm{Fe}_{3} \mathrm{O}_{4}$ nanoparticles exhibiting high heating efficiency in magnetic hyperthermia," The Journal of Physical Chemistry C, vol. 118, no. 16, pp. 86918701, 2014.

[24] S. Palchoudhury, Y. L. Xu, W. An, C. H. Turner, and Y. P. Bao, "Platinum attachments on iron oxide nanoparticle surfaces," Journal of Applied Physics, vol. 107, no. 9, Article ID 09B311, 2010.

[25] Y. L. Xu, S. Palchoudhury, Y. Qin, T. MacHer, and Y. P. Bao, "Make conjugation simple: a facile approach to integrated nanostructures," Langmuir, vol. 28, no. 23, pp. 8767-8772, 2012.

[26] Y. Xu, Y. Qin, S. Palchoudhury, and Y. Bao, "Water-soluble iron oxide nanoparticles with high stability and selective surface functionality," Langmuir, vol. 27, no. 14, pp. 8990-8997, 2011.

[27] J. Mohapatra, A. Mitra, D. Bahadur, and M. Aslam, "Surface controlled synthesis of $\mathrm{MFe}_{2} \mathrm{O}_{4}(\mathrm{M}=\mathrm{Mn}, \mathrm{Fe}, \mathrm{Co}, \mathrm{Ni}$ and $\mathrm{Zn})$ nanoparticles and their magnetic characteristics," CrystEngComm, vol. 15, no. 3, pp. 524-532, 2013.

[28] K. Nakamoto, Infred and Raman Spectra of Inorganic and Coordination Compounds, John Wiley \& Sons, New York, NY, USA, 4th edition, 1986.

[29] H. B. Abrahamson and H. C. Lukaski, "Synthesis and characterization of iron stearate compounds," Journal of Inorganic Biochemistry, vol. 54, no. 2, pp. 115-130, 1994.

[30] M. Darbandi, F. Stromberg, J. Landers et al., "Nanoscale size effect on surface spin canting in iron oxide nanoparticles synthesized by the microemulsion method," Journal of Physics D: Applied Physics, vol. 45, no. 19, Article ID 195001, 2012.

[31] A. Prakash, H. G. Zhu, C. J. Jones et al., "Bilayers as phase transfer agents for nanocrystals prepared in nonpolar solvents," ACS Nano, vol. 3, no. 8, pp. 2139-2146, 2009. 

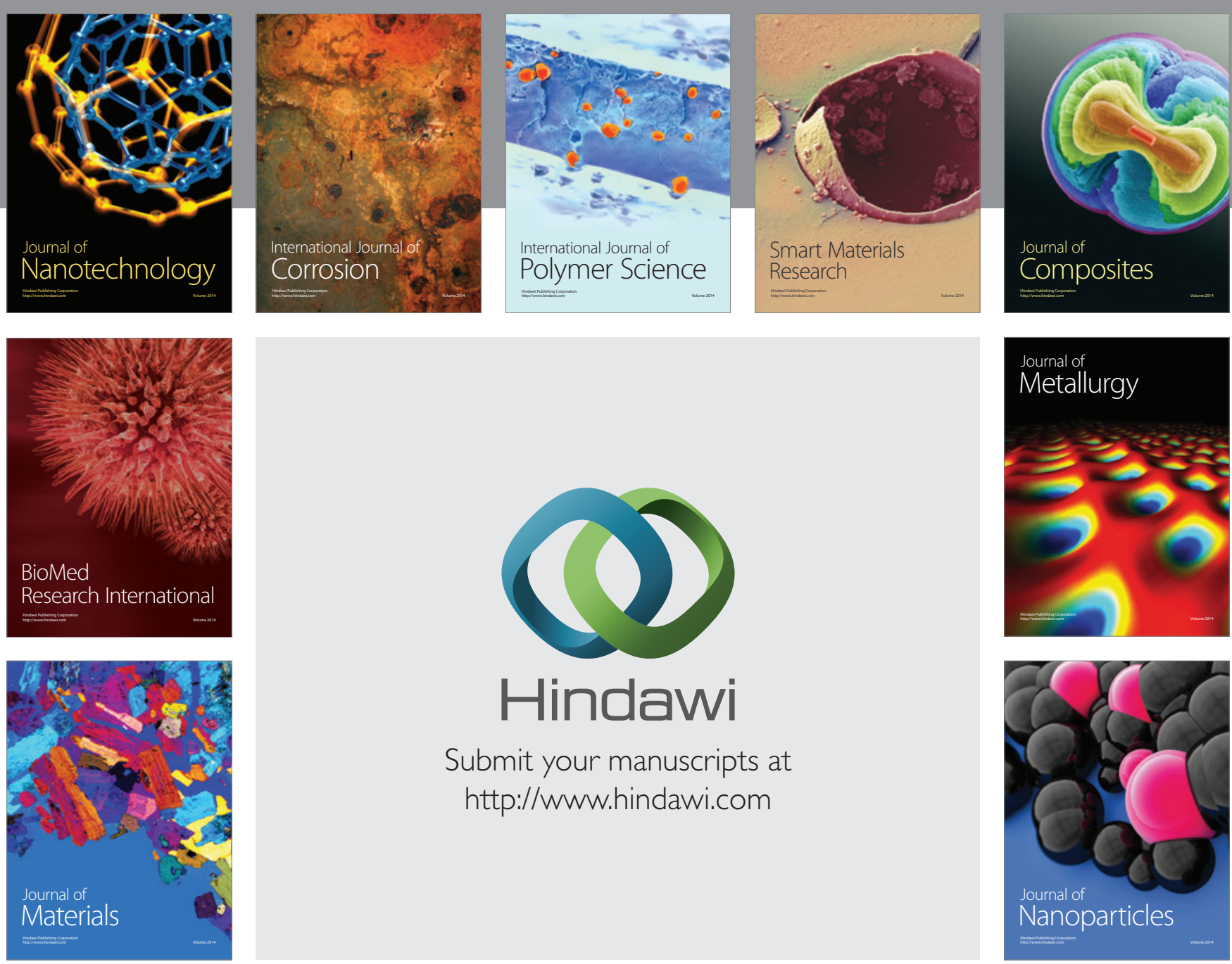

Submit your manuscripts at http://www.hindawi.com
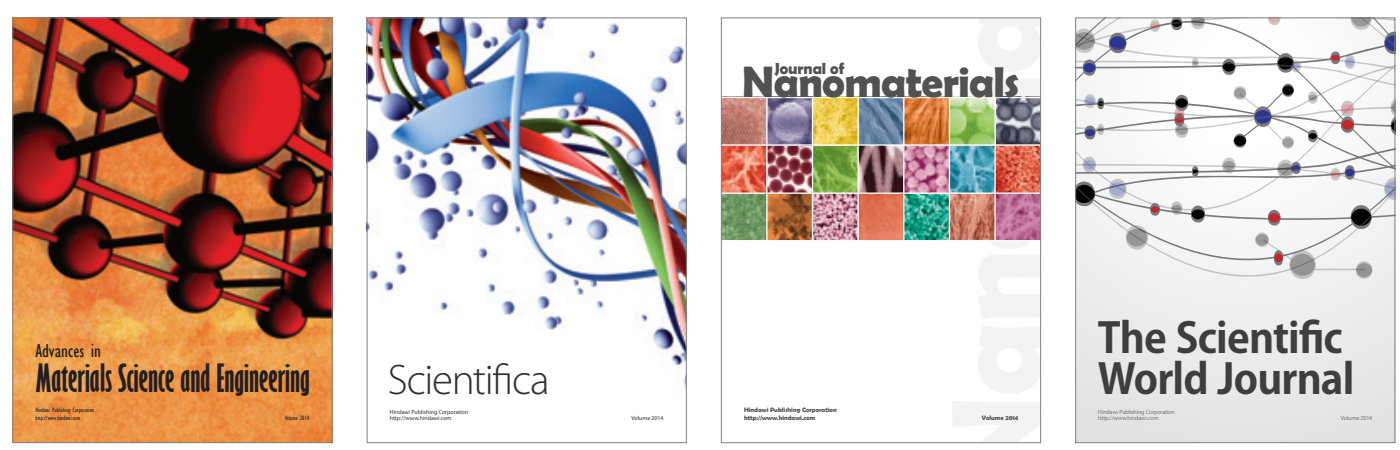

\section{The Scientific World Journal}
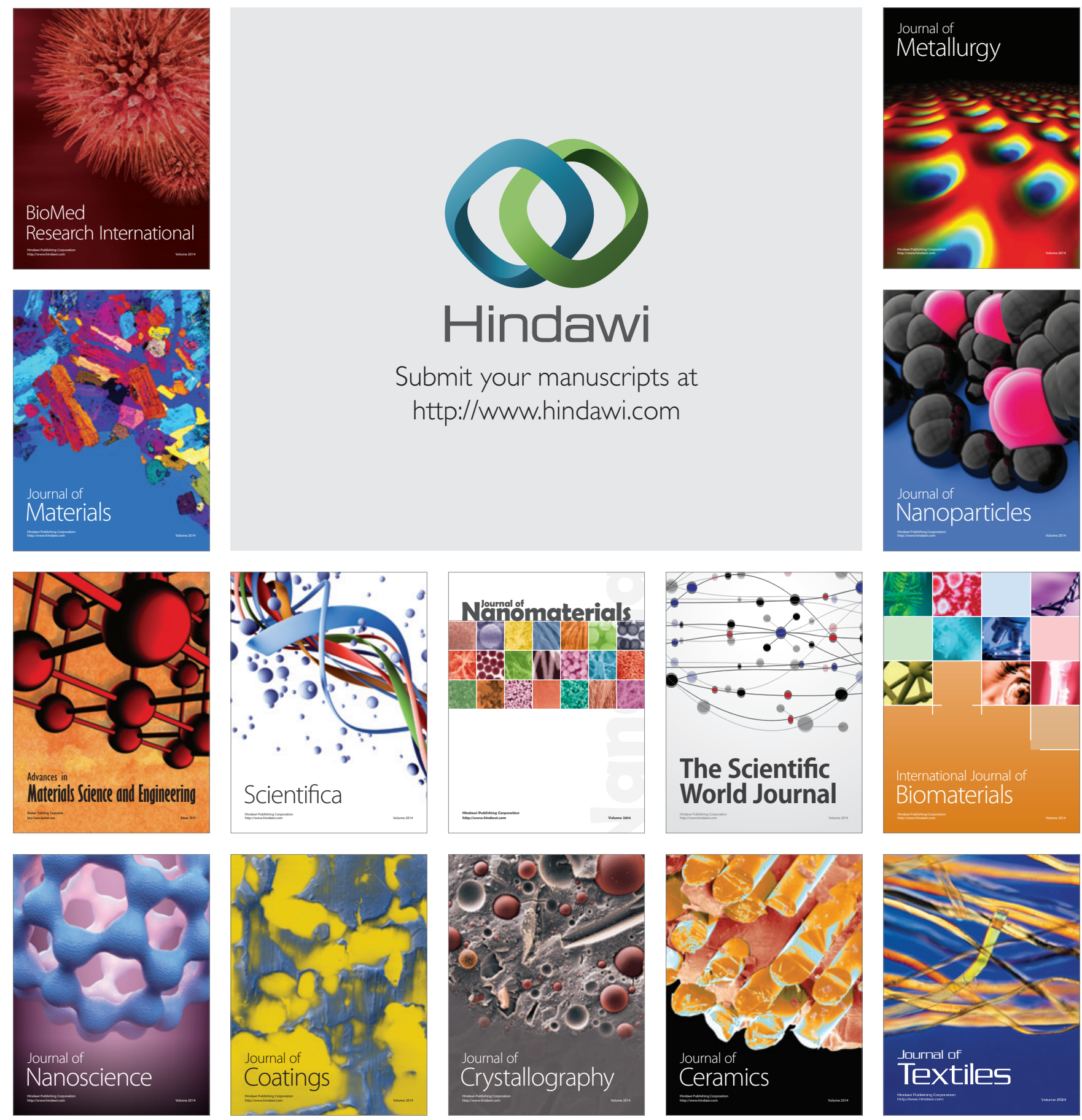